\title{
Bamboo Tissue Culture - Commercial Applications and Environment
}

\author{
Ashish Ranjan* \\ Post Graduate Department of Biotechnology, T. M. Bhagalpur University, \\ Bhagalpur, Bihar, India \\ *Corresponding Author: Ashish Ranjan, Post Graduate Department of \\ Biotechnology, T. M. Bhagalpur University, Bhagalpur, Bihar, India.
}

Received: August 31, 2021

Published: September 15, 2021

(C) All rights are reserved by Ashish Ranjan.
Bamboo is fastest growing multipurpose renewable woody plant on the earth (the highest recorded growth rate is $1 \mathrm{~m} /$ day which makes $1 \mathrm{~mm}$ of growth in every 90 seconds). They are high biomass producing perennial plants belonging to the family Poaceae. It is thought to have originated in China as per the first use of bamboo so far recorded. The genera of bamboo grown today are known to be evolved from primitive grasses around thirty million years ago. Bamboos are distinguished as woody culms, dense and unique rhizomatous system (rendering its fast growth) with gregarious flowering nature. Bamboo vegetation is widely distributed ranging from high altitude of $4000 \mathrm{~m}$ in Himalayas to low altitude areas. Bamboo grows in almost all type of climate (except in extreme cold regions). They are commonly found in evergreen, wet and deciduous (moist and dry) forests. The regions of heavy downpour are also suitable for bamboo growth which is hardly suitable for other useful vegetation. This cosmopolitan distribution of bamboo makes it easily available for mankind.

Now a days bamboos are in demand for more than 3000 commercial applications and uses. These variant uses are due to their natural physical characteristics of being strong, flexible, light smooth, round and hollow stem characteristics. The mechanical orientation due to which they can be split, varied ranges of internodal length, high flexibility and the diversity among the genera make them most useful plants worldwide. Since dawn the civilisation, products such as arrows, building materials, ladders etc. have been recorded in use by mankind. Additionally, they have been in use as food, decorative items, paper, pulp, ornaments and many more. Being versatile group of plants, they are capable of providing ecological, economical as well as source of livelihood to the rural people (hence called "Poor man's timber"). They being renewable source of energy, absorb greenhouse gases, as a suitable substitute of timbers (thereby checking deforestation), prevent soil erosion and the best available natural resources for carbon sequestration are major reasons as why we consider that the bamboos have the significant role in saving our environment and the earth.

\section{Micropropagation of bamboos}

Forest produce of bamboo is now at the threshold of supply due to its increasing utility and demand in our day to day life. We are harvesting millions of bamboos for our daily use but proportionately we do not plant them. Rural people cut hundreds of clumps each year but comparatively less culms are being planted by them. Hence, the growth ratio to harvest ratio is not balanced. This may cause high depletion of bamboo from our natural vegetation.

Quality and mass production is the only method to meet the bamboo demand. Enhanced production includes various techniques of rapid and large scale production. Henceforth, the only reliable option with us is to follow the technique of "clonal micropropagation". The technique deals with the tissue culture of bamboo using various methods i.e. organogenesis and somatic embryogenesis. Organogenesis refers to the development of organs (shoot/root) to form the complete seedling. Somatic embryogenesis on other hand refers to development of embryo from a somatic cell of bamboo culm. Detail stepwise measures of micro propagation will be dealt for some commercially most important species worldwide. 
Some of the important steps include: selection of elite mother bamboo clump, choice of explants (nodal or meristematic), aseptic initiation of cultures, various surface sterilization techniques used, effect of basal medium, additives, carbon source, solidifying agents, pH etc. Effect of seasons, phytohormones, in vitro culture conditions (temperature, humidity, and photoperiod), culture proliferation and hormones for multiplication of microshoots need to be considered for commercial regeneration of bamboo cultures. Synergistic effects of phytohormones have proved to be beneficial in commercial regeneration of bamboo seedlings.

Some of the commercially important species are: Bambusa balcooa, B. tulda, B. nutans, B. bambos, B. pallida, Dendrocalamus asper, D. stocksii, D. hamiltonii, D. brandisii, D. giganteus, Thyrsostachys oliveri, Phyllostachys nigra etc. Those methods have been proved to be beneficial in developing millions of identical germ free plants from a single explant used. The method exploits the "Totipotency" character of the plant cell.

Advantages of micropropagation include: less resources employed, rapid regeneration, large scale production of pathogen free and superior quality traits. Some of the challenges are (endogenous pathogens, hyperhydricity, mortality during hardening etc.) being faced in a production centre and by scientists during micro propagation. However, those bottlenecks have been easily handled now.

\section{Genetic fidelity}

Seedlings raised through organogenesis or somatic embryogenesis are thought to exhibit similar behaviour in terms of growth, thickness, number of culms, pathogen susceptibility etc. Axillary bud proliferations are one of the most widely accepted method of bamboo regeneration (organogenesis) by the researchers around the globe. Somatic cells developed through embryogenesis are of course the copy of mother plant. The culture plants are expected to be genetically identical although variations have been observed despite of growing them in the same media and environment (controlled condition). Dedifferentiation of specialized cells causes more genetic instability. Somaclonal variations are the cause of these genetic differences. It affects our goal of propagating genetically identical plants on large scales. These cause a series of genetic and epigenetic variations. A convergence of plant tissue culture and molecular biology will establish parameters to get desired bamboo tissue culture plants on large scale.

\section{Environmental cleanser}

Being a forest produce with high capacity of biomass regeneration, bamboo has tremendous impact on environment. Notably, they are substitute of timber and thereby, they result in lesser cut down of other scarce vegetative resource, they prevent soil erosion, help in soil conservation, cause conversion of $\mathrm{CO}_{2}$ to $\mathrm{O}_{2}$ and also result in decreasing the effect of global warming.

Carbon accumulation is more by the use of fuels/industrial releases of gases. This results in global warming. To decrease the level of present carbon in atmosphere, a carbon sink is required. This can be done by high vegetative plantation/growth considering time frame as well. Climax forest/vegetation are referred to a vegetation/forest where neither carbon source nor sink is present. Various strategies need to be adopted like conservation (conserve requirement of sustainable bio-resource can be met by bamboo plantation), enhanced regeneration (deforestation practice has reached climax and the immediate demand of wood to meet the threshold can only be met by bamboo plantation.), urban forestry (for the boundaries of the main/cash crops.), peat production (accumulation of partially decayed biodegradable contents which might completely decay).

Role of Bamboo in $\mathrm{CO}_{2}$ sequestration:

- Being fast in growth, bamboo can make a good carbon sink for climax forest and enhanced regeneration.

- Bamboo releases about 35\% more oxygen than a timber plant of equal coverage.

- Bamboo helps in ultraviolet rays to reach less on earth by decreasing the light intensity.

- Bamboo can be planted in strategies mentioned as conservation, urban forestry.

- Cutting down a tree decreases biomass in forest (thinning of forest) while with bamboo case is reversed. Vegetation grows more if we cut down bamboo culms only.

- Bamboo helps in accumulation of carbon above ground, below ground and in also soil pools.

Besides, bamboos are natural and renewable resource of energy. They prevent soil erosion and are able o survive in almost all edaphic conditions. 
Future aspects

Owing to the efficient tools and techniques involved in bamboo tissue culture, commercial production of elite bamboo plants has been feasible in the past few decades. Plant tissue culture techniques are improvised for rapid and industrial production of bamboo seedlings with superior physical traits, culm girth and higher secondary metabolites production. The scope of research and production can lead to vast opportunities of employment from small scale to high end value products of bamboo. The cleansing of environment will fall as gratuity of the scientific work. BioCNG, Bioethanol, Biodiesel production from bamboo will tackle various upcoming challenges for the Govt. of our country. Further, in vitro propagation for selective metabolite production is found highly useful for secondary metabolites and medicinally important compounds.

Volume 5 Issue 10 October 2021

(c) All rights are reserved by Ashish Ranjan. 